\title{
High-bandwidth and low-loss multimode polymer waveguides and waveguide components for high- speed board-level optical interconnects
}

N. Bamiedakis, J. Chen, R. V. Penty, I. H. White

N. Bamiedakis, J. Chen, R. V. Penty, I. H. White, "High-bandwidth and lowloss multimode polymer waveguides and waveguide components for highspeed board-level optical interconnects," Proc. SPIE 9753, Optical Interconnects XVI, 975304 (15 March 2016); doi: 10.1117/12.2212546

SPIE. Event: SPIE OPTO, 2016, San Francisco, California, United States 


\title{
High-bandwidth and low-loss multimode polymer waveguides and waveguide components for high-speed board-level optical interconnects
}

\author{
N. Bamiedakis*, J. Chen, R.V. Penty, I. H. White \\ Electrical Engineering Division, Engineering Department, University of Cambridge, \\ 9 J. J. Thomson Avenue, Cambridge, CB3 OFA, UK \\ *nb301@ cam.ac.uk
}

\begin{abstract}
Multimode polymer waveguides are being increasingly considered for use in short-reach board-level optical interconnects as they exhibit favourable optical properties and allow direct integration onto standard PCBs with conventional methods of the electronics industry. Siloxane-based multimode waveguides have been demonstrated with excellent optical transmission performance, while a wide range of passive waveguide components that offer routing flexibility and enable the implementation of complex on-board interconnection architectures has been reported. In recent work, we have demonstrated that these polymer waveguides can exhibit very high bandwidth-length products in excess of $30 \mathrm{GHz} \times \mathrm{m}$ despite their highly-multimoded nature, while it has been shown that even larger values of $>60 \mathrm{GHz} \times \mathrm{m}$ can be achieved by adjusting their refractive index profile. Furthermore, the combination of refractive index engineering and launch conditioning schemes can ensure high bandwidth ( $>100 \mathrm{GHz} \times \mathrm{m})$ and high coupling efficiency $(<1 \mathrm{~dB})$ with standard multimode fibre inputs with relatively large alignment tolerances $\left(\sim 17 \times 15 \mu \mathrm{m}^{2}\right)$. In the work presented here, we investigate the effects of refractive index engineering on the performance of passive waveguide components (crossings, bends) and provide suitable design rules for their on-board use. It is shown that, depending on the interconnection layout and link requirements, appropriate choice of refractive index profile can provide enhanced component performance, ensuring low loss interconnection and adequate link bandwidth. The results highlight the strong potential of this versatile optical technology for the formation of high-performance board-level optical interconnects with high routing flexibility.

Keywords: optical interconnects, polymer waveguides, waveguide components, waveguide bandwidth
\end{abstract}

\section{INTRODUCTION}

Optical interconnection technologies are being increasingly considered for use within high-performance electronic systems such as data servers, memory storage systems and supercomputers to address the foreseen bottleneck in system performance due to the inherent limitations of copper-based interconnects [1-3]. Optical interconnects offer larger bandwidth, reduced power consumption, immunity to electromagnetic interference and relaxed thermal management requirements. Optical links are already widely deployed for short-reach rack-to-rack communication in data centres, while multimode fibre links have been recently introduced in electrical drawers for intra-rack communication in supercomputer systems [4, 5]. The further integration of optical links onto low-cost printed circuit boards (PCBs) can offer even larger interconnection densities and enable much higher interconnection capacities in such systems. As a result, recent research has focused on the development of waveguide-based optical technologies that enable the formation of cost-effective optical backplanes and board-level optical interconnections. In particular, polymer multimode waveguides are considered to be as an attractive technology as the polymer materials allow direct integration onto PCBs, while their large waveguide dimensions (typically 30 to $70 \mu \mathrm{m}$ ) allow system assembly with common pick-and-place tools [6-8]. In recent years, a large number of system demonstrators featuring multimode polymer waveguides have been realized, showcasing the potential of this technology [9-11].

Optical Interconnects XVI, edited by Henning Schröder, Ray T. Chen, Proc. of SPIE Vol. 9753, 975304

(C) 2016 SPIE $\cdot$ CCC code: 0277-786X/16/\$18 · doi: 10.1117/12.2212546 
Our work at the University of Cambridge has comprised the study of the optical transmission properties of siloxanebased multimode waveguides and passive waveguide components [12-14], the development of methods enabling their cost-effective integration onto PCBs [6] and the realization of various backplane demonstrators [11, 15]. Recent work has focused on the potential of using refractive index (RI) engineering in such waveguides, studying the loss and bandwidth performance of waveguides and waveguide components with different RI profiles. These studies reveal that these multimode polymer waveguides exhibit very high bandwidth-length products (BLP) > $30 \mathrm{GHz} \times \mathrm{m}$ despite their highly-multimoded nature and indicate that even higher bandwidth $>100 \mathrm{GHz} \times \mathrm{m}$ can be achieved with appropriate refractive index engineering and launch conditioning schemes. The studies on the transmission properties of passive waveguide components, such as waveguide bends and crossings that are the building blocks of any interconnection layout, show that RI engineering can enable optimized loss performance in complex waveguide layouts while ensuring adequate link bandwidth. In the sections that follow, our recent results on waveguide bandwidth are presented and the studies on the performance of the waveguide components are reported.

\section{MULTIMODE POLYMER WAVEGUIDE TECHNOLOGY}

This particular waveguide technology is based on the use of siloxane polymer materials developed by Dow Corning. These materials have been appropriately engineered to meet the requirements of the specific application: they exhibit low loss at the datacommunications' wavelength range, they can withstand the high-temperature environments required in PCB manufacturing (solder reflow, board lamination), they are easily processed and patterned and they exhibit long lifetimes and stability in the typical operating environment of electronic systems $[11,16,17]$. The materials can be deposited by various methods (doctor-blading, spin coating or drop casting) on any type of rigid substrate (FR4, silicon, glass) and allow waveguide patterning with conventional mask aligners, direct laser writing systems or embossing tools. Moreover, they enable the formation of flexible samples with similar performance metrics as on rigid substrates [17]. The refractive index (RI) difference $\Delta \mathrm{n}$ between the core and cladding material is nominally $\sim 0.02$ at $850 \mathrm{~nm}$ but it can be tuned appropriately to adjust the RI profile in the waveguide core [18]. The waveguide RI profile can be modified by controlling the fabrication parameters, resulting in varied RI profiles from step-index (SI) to graded-index (GI). The typical dimensions and pitch of the fabricated waveguides are $\sim 50 \mu \mathrm{m}$ and $250 \mu \mathrm{m}$ respectively, in order to match multimode fibres (MMFs) and ribbons and vertical-cavity surface-emitting laser (VCSEL) arrays. Such waveguide dimensions provide $1 \mathrm{~dB}$ alignment tolerances of $\sim \pm 10 \mu \mathrm{m}$ for MMF inputs and butt-coupled VCSEL sources enabling

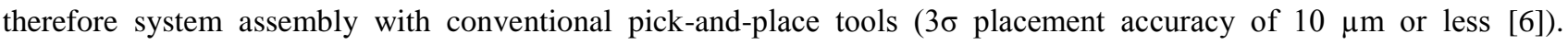
Waveguides with different dimensions and pitches can also be readily formed.

This waveguide technology primarily targets the $850 \mathrm{~nm}$ wavelength range where low-cost multimode VCSEL sources are available. These lasers are the preferred sources in these applications as they are sufficiently low-cost, exhibit very low threshold currents $(\sim 1 \mathrm{~mA})$ and don't require any temperature control resulting thus in low power consumption, emit relatively large optical power (>1 mW), exhibit large bandwidth (>20 GHz) and can be readily formed in large one- or two-dimensional arrays [19]. In recent years, there has been a continuous improvement in the high-speed performance of VCSEL devices, with most recent reports demonstrating $57 \mathrm{~Gb} / \mathrm{s}$ [20], $64 \mathrm{~Gb} / \mathrm{s}$ [21] and $71 \mathrm{~Gb} / \mathrm{s}$ [22] operation. As a result, important questions arise on the potential of this waveguide technology to support such high data rates because of their highly-multimoded nature. To address this issue, we have recently demonstrated $25 \mathrm{~Gb} / \mathrm{s}$ [23] and $40 \mathrm{~Gb} / \mathrm{s}$ [24] data transmission over $1.4 \mathrm{~m}$ and $1 \mathrm{~m}$ long spiral waveguides respectively, and we have presented bandwidth studies based on frequency response measurements on long spiral waveguides under different launch conditions [25]. The frequency response studies have shown that these waveguides exhibit bandwidth-length products (BLP) > $35 \mathrm{GHz} \times \mathrm{m}$, while the data transmission experiments have demonstrated that, for $40 \mathrm{~Gb} / \mathrm{s}$ data transmission, power rather than bandwidth is the limiting factor in the maximum reach of these waveguide links. In order to further explore the potential of the waveguides, time domain measurements have been undertaken on long spiral waveguides to identify their bandwidth. 


\section{DISPERSION STUDIES}

The dispersion studies are carried out on $1 \mathrm{~m}$ long spiral waveguides and are based on the comparison of the width of a short optical pulse before and after transmission over the multimode waveguide. For these studies, two waveguide samples with a different RI profile are employed in order to assess its effect on bandwidth performance. The samples are fabricated on an 8-inch Si substrate with conventional photolithography. Both samples have similar core size $(\sim 32 \times 35$ $\mu \mathrm{m}^{2}$ ), but the process parameters are slightly adjusted so as to result in a slightly different RI profile for the two samples. Their RI profile are measured using the near field refractive index method and are shown in Figure 1(a). The first sample has a more uniform profile over the waveguide core, whilst the second clearly exhibits a triangular area of higher index towards the top side of the waveguide core. The two samples are denoted as "SI" and "GI" respectively in the rest of the paper, although their RI profiles do not match the typical SI and GI profiles encountered in glass fibres. The input and output facets of the waveguide samples are exposed with a dicing saw, while no polishing steps are undertaken to improve the quality of the facets produced. An image of the structure of the spiral waveguide illuminated with red light is shown in Figure 1(b), while Figure 1(c) shows near field images of the waveguide output facet of the two samples at $850 \mathrm{~nm}$.

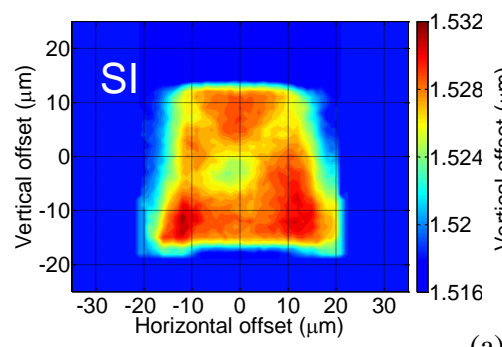

(a)

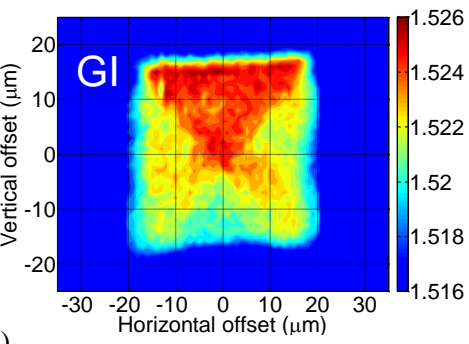

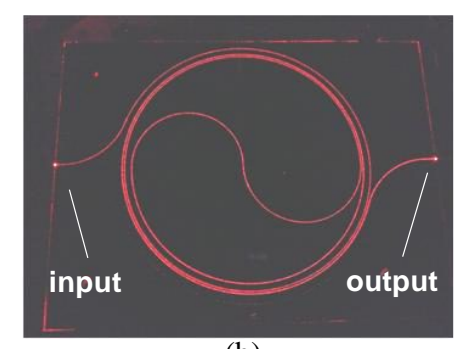

(b)

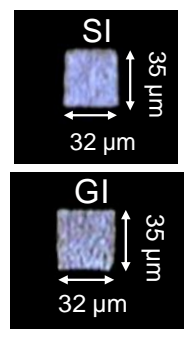

(c)

Figure 1. (a) Measured RI profile at $678 \mathrm{~nm}$ for the two waveguide samples and images of (b) the GI spiral waveguide illuminated with red light and (c) the output facet of the two waveguides at $850 \mathrm{~nm}$.

The dispersion measurements are carried out with a 50/125 $\mu \mathrm{m}$ OM3 MMF input so as to emulate a launch condition likely to be encountered in a real-world system. Moreover, the experiments are repeated when a mode mixer (MM Newport FM-1) is employed at the MMF input in order to further investigate the waveguide bandwidth under a relatively more overfilled excitation. The MM generates a more uniform mode power distribution inside the input MMF resulting therefore, in a larger percentage of power coupled to higher order modes at the waveguide input. As a result, larger intermodal dispersion is expected at the waveguide output and lower waveguide bandwidth for such an excitation. The experimental configurations used in the pulse broadening measurements are illustrated in Figure 2. A femtosecond Ti:Sapphire laser operating at $850 \mathrm{~nm}$ is used as the short pulse source [ 200 fs full-width-at-half-maximum (FWHM)], and a FR103-MN autocorrelator as the detector. The emitted light is coupled to a 50/125 $\mu \mathrm{m}$ OM3 MMF patchcord using a pair of microscope objectives. For the "overfilled" launch, the MM is inserted at the waveguide input. The other end of the MMF patchcord is cleaved and positioned on a micro-controlled translation stage and is butt-coupled to the waveguide input. Figure 2(c) and (d) show images of the near field images of the MMF output with and without the use of the MM. A displacement sensor is employed to measure the input offset and enable the control of the launch position. At the waveguide output, a 16× (NA of 0.32) microscope objective is used to collect the transmitted light and deliver it to the autocorrelator. The insertion loss of both samples is found to be in the range 7 to $8 \mathrm{~dB}$. 


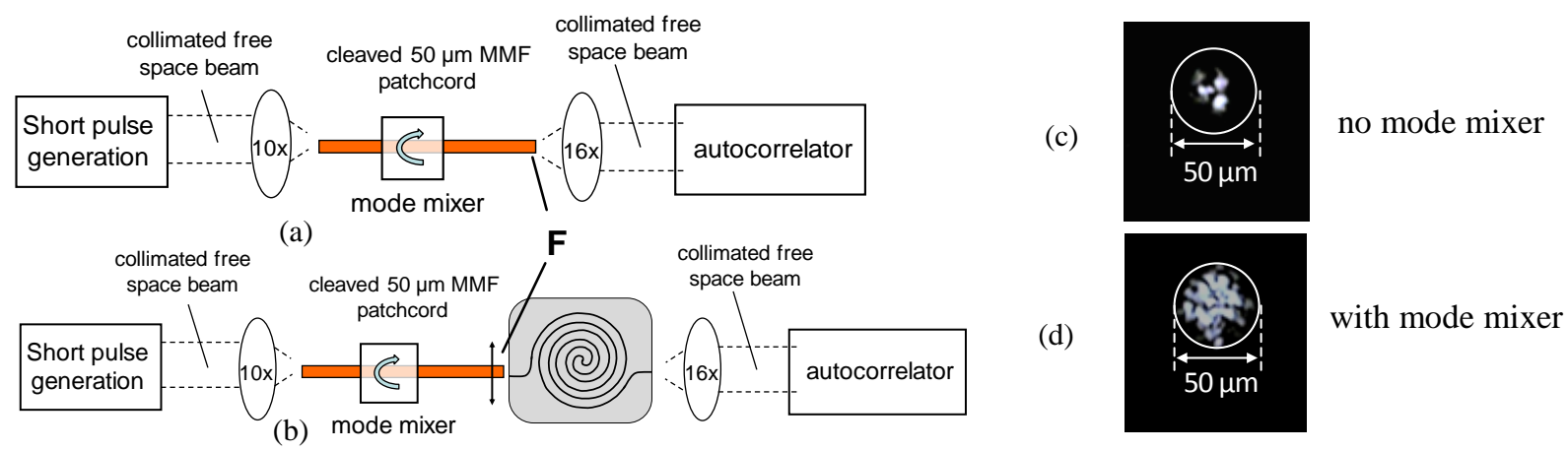

Figure 2. Experimental setup used in pulse broadening measurements: (a) back-to-back and (b) waveguide link, and near-field images of the MMF cleaved end [point F in (a) and (b)] (c) without and (d) with the use of the mode mixer.

The autocorrelation traces of the received pulse are recorded for the different input positions. The shape of the received pulse is approximated using curve-fitting and standard functions ( $\mathrm{sech}^{2}$, Gaussian, Lorentzian) allowing the estimation of the pulse width at the waveguide output. A similar setup and methodology is used for the back-to-back link (without the waveguide) in order to obtain the input pulse shape and pulse width. The frequency response of the waveguide, and therefore its $3 \mathrm{~dB}$ bandwidth (BW), is found by comparing the Fourier transform of the received pulses for the waveguide and back-to-back link. Figure 3 shows the estimated $3 \mathrm{~dB}$ waveguide bandwidth for the two spiral waveguides with and without the use of the MM.
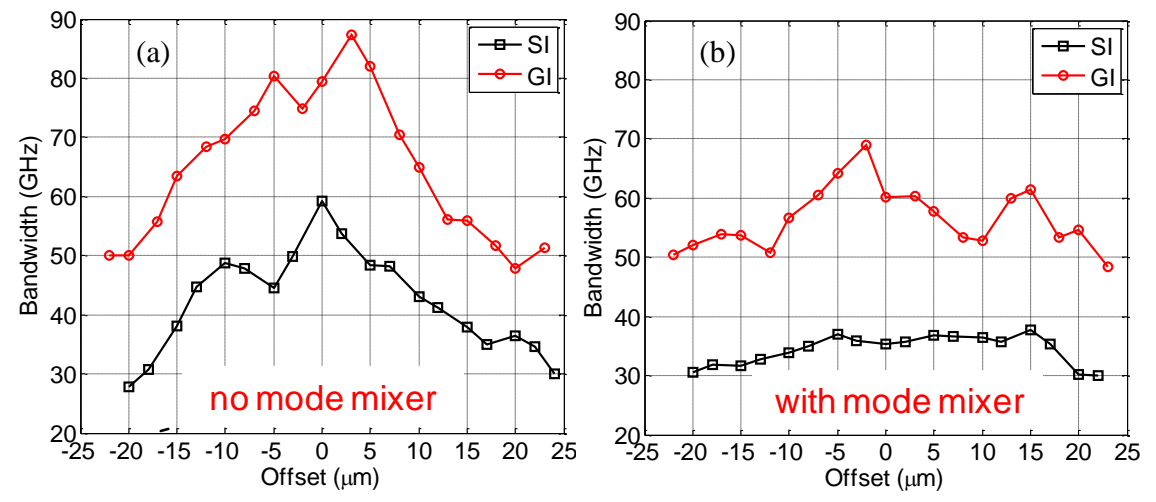

\begin{tabular}{|c|c|c|}
\hline $\begin{array}{c}\text { Launch condition } \\
\text { and offset range }\end{array}$ & $\begin{array}{c}\mathrm{SI} \\
\mathrm{GHz} \times \mathrm{m}\end{array}$ & $\begin{array}{c}\mathrm{Gl} \\
\mathrm{GHz} \times \mathrm{m}\end{array}$ \\
\hline $\begin{array}{c}\text { No MM } \\
-15 \mu \mathrm{m} \leq \Delta \mathrm{x} \leq+10 \mu \mathrm{m}\end{array}$ & $>40$ & $>60$ \\
\hline $\begin{array}{c}\text { With MM } \\
-20 \mu \mathrm{m} \leq \Delta \mathrm{x} \leq+20 \mu \mathrm{m}\end{array}$ & $>30$ & $>50$ \\
\hline
\end{tabular}

(c)

Figure 3. Calculated $3 \mathrm{~dB}$ waveguide bandwidth for the SI and GI $1 \mathrm{~m}$ long spiral waveguides under a $50 \mu \mathrm{m}$ MMF launch (a) without and (b) with the use of a MM at the waveguide input. (c) Summary of BW results.

The results indicate that both waveguide samples exhibit a BLP larger than $30 \mathrm{GHz} \times \mathrm{m}$ even when a mode mixer is used at the waveguide input. As expected, the use of the mode mixer results in lower BLP values but in a smaller variation across input offsets owing to the more uniform mode power distribution at the waveguide input. Large input offsets result in larger percentage of power coupled to higher order modes at the waveguide input, and therefore, in larger intermodal dispersion and lower waveguide bandwidth. The GI waveguide exhibits larger BLP than the SI sample with BLP values recorded larger than $50 \mathrm{GHz} \times \mathrm{m}$ for all offsets and both launch conditions. When no MM is employed at the GI waveguide input, very high BW values in the range 50 to $85 \mathrm{GHz} \times \mathrm{m}$ are obtained. Figure 3(c) summarises the offset range for each sample that ensures that a particular waveguide BLP value is achieved. It is found that alignment tolerances larger than $\pm 10 \mu \mathrm{m}$ are obtained for both samples, indicating that high-speed transmission over these waveguides is relatively insensitive to input offsets. The asymmetry observed with respect to the offset direction (+/-) is due to the spiral shape of the waveguide. Finally, it should be noted that the large BLP values $>60 \mathrm{GHz} \times \mathrm{m}$ obtained for the GI sample indicate the potential to achieve $100 \mathrm{~Gb} / \mathrm{s}$ transmission over a single waveguide channel. 


\section{LAUNCH CONDITIONING}

The dispersion studies presented above indicate that very high BLPs in excess of $60 \mathrm{GHz} \times \mathrm{m}$ can be obtained in GI waveguides, and therefore the potential to achieve $100 \mathrm{~Gb} / \mathrm{s}$ transmission over a single channel. For such a high-speed link however, it is imperative to ensure that both adequate waveguide bandwidth and received optical power are available with relaxed tolerances. As a result, we explore the potential of using launch conditioning schemes at the waveguide input in order to achieve $100 \mathrm{~Gb} / \mathrm{s}$ over a $1 \mathrm{~m}$ long polymer waveguide. Such launch conditioning schemes (e.g. offset launch) have thoroughly been studied and implemented in the context of MMFs enabling the extension of the reach of MMF links and the transmission of higher data rates [26].

For these studies, we employ shorter reference waveguides [schematic shown in Figure 4(a), length $19.2 \mathrm{~cm}$ ] and a restricted launch which is implemented at the waveguide input with a $10 \times$ microscope objective. The input spot has a 5 $\mu \mathrm{m}$ FWHM. The experimental setup is shown in Figure 4(b). For these measurements, a femtosecond erbium-doped fibre laser (TOPTICA FSS) emitting at $1574 \mathrm{~nm}$ is employed as the short pulse ( 400 fs FWHM) laser source while a frequency-doubling crystal (MSHG1550-0.5-1) is used to halve the wavelength to $787 \mathrm{~nm}$, so as to investigate the waveguide bandwidth at a wavelength close to the $850 \mathrm{~nm}$ range of interest. A matching autocorrelator is employed at the waveguide output. Both the power received at the waveguide output and the autocorrelation traces of the received pulses are recorded as the launch position is offset in both the vertical and horizontal directions. The insertion loss of the waveguide is found to be $\sim 1.5 \mathrm{~dB}$. The obtained waveguide BLP and the normalised received optical power are plotted in Figure 4(c) and (d) respectively as a function of the input position.

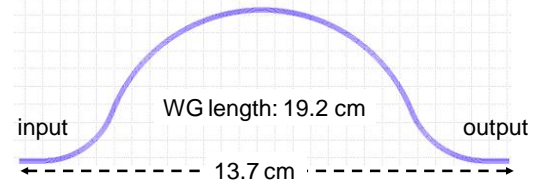

(a)

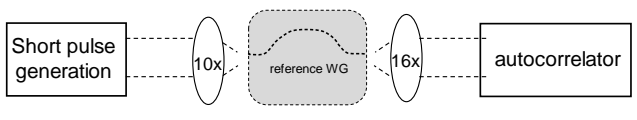

(b)

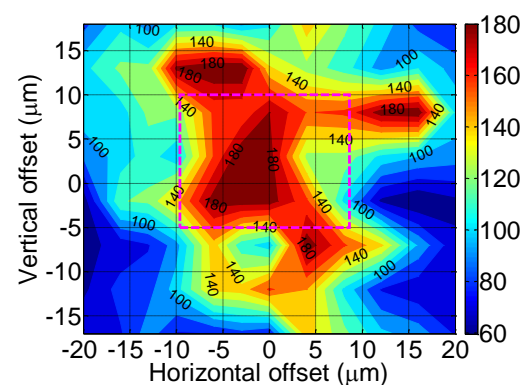

(c)

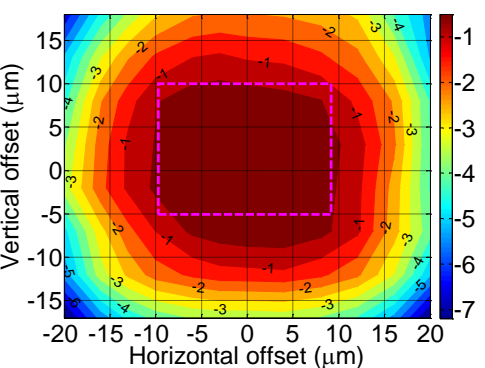

(d)

Figure 4. Schematic of (a) the reference waveguide and (b) the experimental setup, and measured (c) BLP (in GHz $\times$ m) and

(d) normalised received power (in $\mathrm{dB}$ ) for the GI reference waveguide as a function of the launch position. The pink rectangle denotes the range of input offsets that ensures both a normalized coupling loss $<1 \mathrm{~dB}$ and a BLP $>100 \mathrm{GHz} \times \mathrm{m}$.

The results indicate that there is a relatively large range of input offsets that ensures that the BLP value is larger than 100 GHz $\times \mathrm{m}\left(\sim 18 \times 20 \mu \mathrm{m}^{2}\right)$. If the coupling loss is considered, a range of $\sim 17 \times 15 \mu \mathrm{m}^{2}$ is identified that also ensures that any additional loss due to alignment offset is below $1 \mathrm{~dB}$ [region delimited with pink region in Figure 4(c) and (d)]. The results indicate that using a launch conditioning scheme that generates a relatively small input spot positioned within this $17 \times 15 \mu \mathrm{m}^{2}$ area at the waveguide input can provide both large bandwidth $(>100 \mathrm{GHz} \times \mathrm{m})$ and low coupling loss $(<1$ $\mathrm{dB}$ ), enabling therefore very high data transmission rates over such multimode waveguides. 


\section{MULTIMODE WAVEGUIDE COMPONENTS}

A wide range of multimode passive waveguide components based on this polymer waveguide technology, such as waveguide bends, crossings, splitters and combiners, have been reported and excellent transmission properties have been demonstrated [12, 14, 27]. These waveguide components form the building blocks of any on-board interconnection architecture and have already been deployed in various optical backplane demonstrators [11, 15]. For example, the terabit-capacity 10-card passive optical backplane reported in [11], exhibits 1800 waveguide crossings and $10090^{\circ}$ bends. This particular waveguide layout was designed to support $10 \mathrm{~Gb} / \mathrm{s}$ data transmission per waveguide channel. In order however, to transmit higher data rates $(25 \mathrm{~Gb} / \mathrm{s}$ or higher) over each waveguide link, the related power budget requirements need to be satisfied and adequate bandwidth need to be available in each waveguide link for the launch conditions likely to be used in a real-world system. As a result, in this work, we present loss and bandwidth studies on the basic waveguide components $\left(90^{\circ}\right.$ bends and $90^{\circ}$ crossings) and study to use of RI engineering in order to optimise the total loss of complex optical paths. These two components (bends and crossings) in particular, exhibit a contrasting behavior with respect to the RI difference $\Delta \mathrm{n}$ between the core and cladding materials: bending loss benefits from stronger optical confinement in the waveguide core (larger $\Delta \mathrm{n}$ values), while waveguide crossings with lower $\Delta \mathrm{n}$ exhibit lower loss per crossing due to the reduced field expansion at the waveguide intersection. A design trade-off therefore exists.

For these studies, 3 waveguide samples with slightly different RI profiles are employed. All samples are fabricated on an 8-inch Si substrate with standard photolithographic processes and comprise a number of different components: (i) waveguides with two $90^{\circ}$ bends with a varying radius of curvature, (ii) waveguides with a varying number of $90^{\circ}$ crossings and (iii) reference waveguides. The reference waveguides have a similar structure and length as the other two components and are used as control samples. Schematics of the waveguide components are shown in Figure 5(a)-(c), while Figure 5(c) and (d) show the measured RI profiles of the 3 waveguide samples (denoted as WG_A, WG_B, WG_C) and their key parameters (size, $\left.\Delta \mathrm{n}_{\max }\right)$.
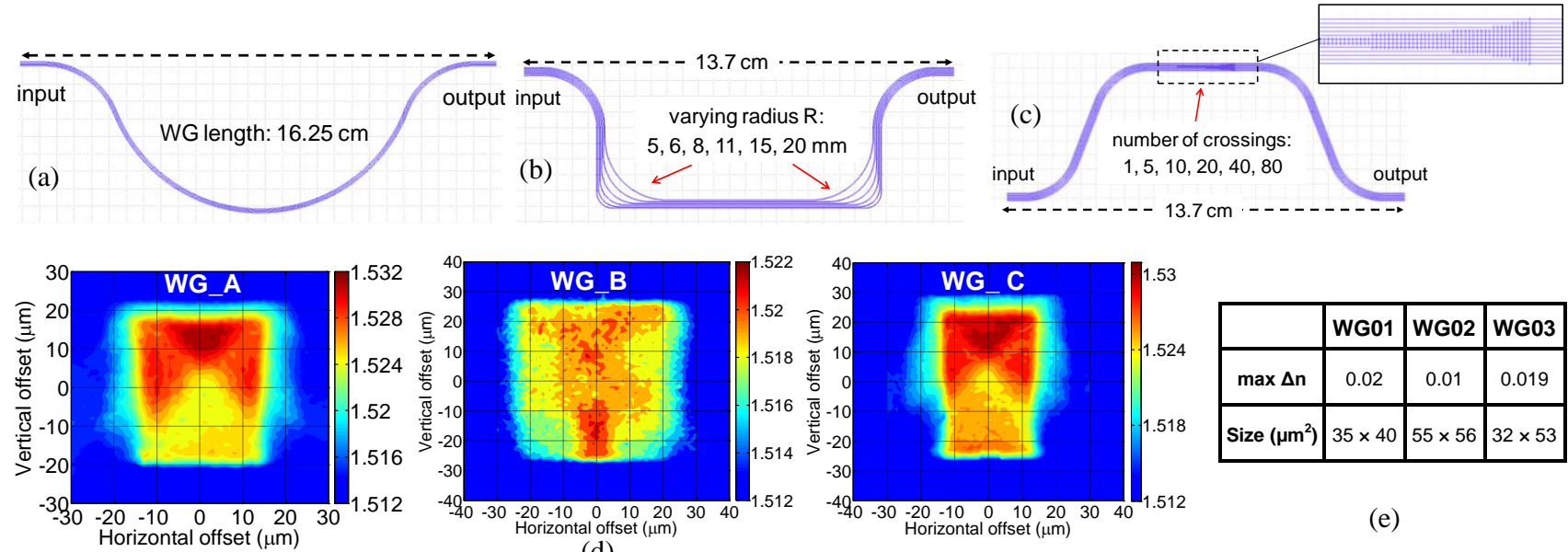

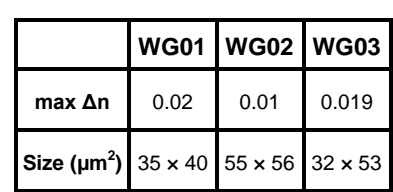

(e)

Figure 5. Schematic of the waveguide components studied: (a) ) reference waveguides, (b) $90^{\circ}$ bends, and (c) $90^{\circ} \mathrm{crossings}$ and (d) measured RI profile of the three waveguide samples at $678 \mathrm{~m}$ and (e) summary of their key parameters.

The insertion loss of all components is measured under a restricted launch using a 9/125 $\mu \mathrm{m}$ SMF input and a more overfilled launch using a 50/125 $\mu \mathrm{m}$ MMF input. The use of the MMF input is expected to result in higher insertion loss values as a larger percentage of power is coupled at the waveguide input to higher order modes, which are more susceptible to radiation losses along the waveguide bends and at the crossing intersections. Moreover, the bandwidth of the reference waveguides is also measured for a $50 \mu \mathrm{m}$ MMF input using the same setup as described in section 2 and shown in Figure 2(b). The obtained insertion loss values and BLP for the reference waveguides are shown in Figure 6. It 
can be noticed that WG_B exhibits the highest insertion loss for a $50 \mu \mathrm{m}$ MMF input, whereas similar values are obtained from all samples for a SMF input. This can be attributed to the low coupling loss and the excitation of lower order modes at the waveguide input due to the small input spot. In terms of bandwidth, WG_B exhibits a much larger BLP value than the two other samples $(\sim 120 \mathrm{GHz} \times \mathrm{m}$ vs $\sim 50 \mathrm{GHz} \times \mathrm{m})$ owing to its lower $\Delta \mathrm{n}$.

(a)

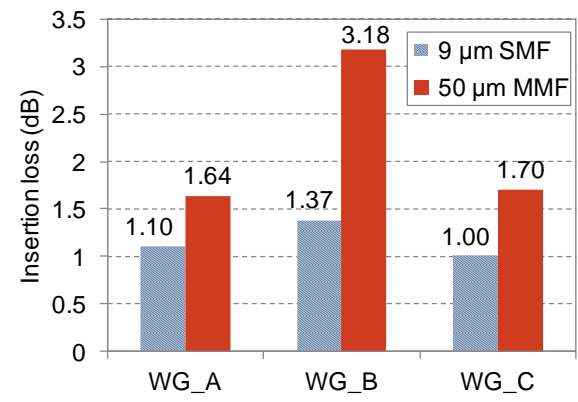

(b)

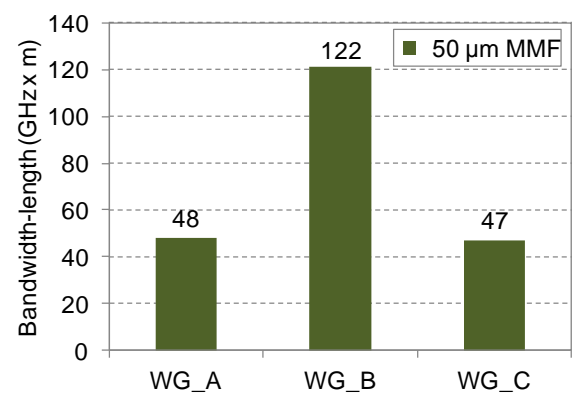

Figure 6. Insertion loss for the reference waveguides for the 3 waveguide samples for a SMF and $50 \mu \mathrm{m}$ MMF input and calculated BLP value for a $50 \mu \mathrm{m}$ MMF input.

The bending and crossing loss of the waveguide components are obtained by subtracting the insertion loss of the respective reference waveguides from the insertion loss of the components recorded under the same launch condition. The obtained loss difference represents therefore the excess loss induced due to the waveguide bends and crossings. The results are shown in Figure 7(a) and 7(b), while Figure 7(c) summarises the loss per crossing observed for each waveguide sample and launch condition. The results demonstrate the WG_B exhibits the highest bending loss due to its large width $(\sim 55 \mu \mathrm{m})$ and lowest $\Delta \mathrm{n}(\sim 0.01)$, while similar loss values are obtained for the other two samples. A low bending loss $<1 \mathrm{~dB}$ is achieved for both samples WG_A and WG_C for radii $>6 \mathrm{~mm}$ for both inputs. The use of the 50 $\mu \mathrm{m}$ MMF results in slightly increased insertion loss values (by $\sim 1 \mathrm{~dB}$ ) due to the higher coupling and bending loss incurred. For the waveguide crossings on the contrary, samples WG_B exhibit the lowest loss per crossing ( $\sim 0.007$ $\mathrm{dB} /$ crossing and $0.02 \mathrm{~dB} /$ crossing for a SMF and MMF input respectively) due to their lower $\Delta \mathrm{n}$ value.

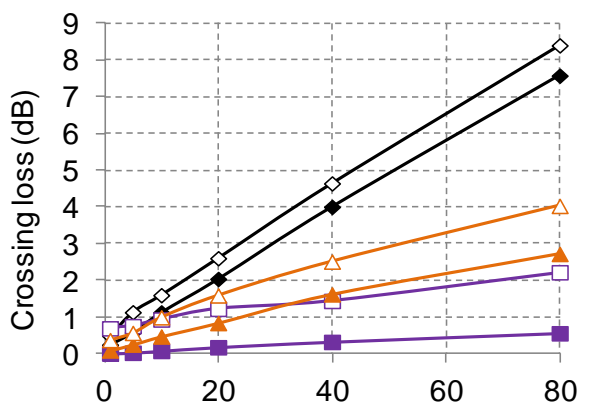

(a) Number of crossings

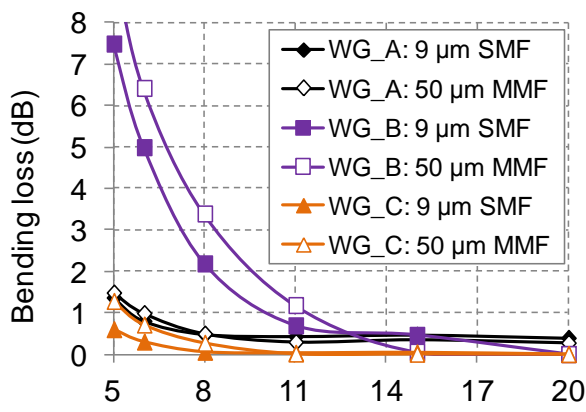

(b) Radius (mm)

\begin{tabular}{|c|c|c|c|}
\hline \multirow{2}{*}{ Input } & \multicolumn{3}{|c|}{ Loss (dB/crossing) } \\
\cline { 2 - 4 } & WG_A & WG_B & WG_C \\
\hline SMF & 0.093 & 0.007 & 0.033 \\
\hline $50 \mu \mathrm{m}$ MMF & 0.098 & 0.019 & 0.046 \\
\hline
\end{tabular}

(c)

Figure 7. Loss of the waveguide (a) crossings and (b) bends, and (c) summary of the loss per crossing for each sample.

The demonstrated waveguide components exhibit excellent optical transmission properties while the performance tradeoff with respect to their RI profile is demonstrated. The insertion loss of a complex waveguide layout that features a number of passive waveguide components can therefore be optimized for a particular launch condition. The index difference $\Delta \mathrm{n}$ can be appropriately chosen to minimize the total insertion loss for a particular waveguide link, while ensuring that adequate bandwidth is available for high-speed operation. Given the tight power budget requirements in high-speed optical links, this optimization becomes particular important for the design of next-generation passive optical backplanes. 


\section{CONCLUSIONS}

In this article, we present recent studies on multimode polymer waveguides and waveguide components and demonstrate that this particular technology can offer low loss transmission and high bandwidth in board-level optical interconnects. The waveguides can exhibit bandwidth-length product values in excess of $60 \mathrm{GHz} \times \mathrm{m}$ for a range of offsets larger than $\pm 10 \mu \mathrm{m}$, while it is shown that even larger BLP values $>100 \mathrm{GHz} \times \mathrm{m}$ can be obtained with relaxed alignment tolerances when appropriate launch conditioning schemes are employed. Finally, studies on waveguide components demonstrate excellent optical transmission properties from these components and indicate that RI engineering can enable optimization of the loss and bandwidth performance of complex waveguide paths. These results highlight the strong potential of this technology in forming cost-effective high-speed board-level optical interconnects and passive optical backplanes.

\section{ACKNOWLEDGEMENTS}

The authors would like to acknowledge Dow Corning for the provision of the polymer samples and the UK EPSRC for supporting this work. Additional data related to this publication is available at the University of Cambridge data repository (https://www.repository.cam.ac.uk/handle/1810/253542).

\section{REFERENCES}

[1]. D. Miller, "Device Requirements for Optical Interconnects to Silicon Chips," Proceedings of the IEEE, vol. 97, pp. 1166-1185, 2009.

[2]. D. W. Huang, T. Sze, A. Landin, R. Lytel, and H. L. Davidson, "Optical interconnects: Out of the box forever," IEEE Journal of Selected Topics in Quantum Electronics, vol. 9, pp. 614-623, 2003.

[3]. C. Hoyeol, P. Kapur, and K. C. Saraswat, "Power comparison between high-speed electrical and optical interconnects for interchip communication," Journal of Lightwave Technology, vol. 22, pp. 2021-2033, 2004.

[4]. A. F. Benner, et al., "Optics for high-performance servers and supercomputers," in Optical Fiber Communication Conference (OFC), pp. 1-3, 2010.

[5]. J. A. Kash, et al., "Optical interconnects in future servers," in Optical Fiber Communication Conference (OFC), pp. $1-3,2011$.

[6]. N. Bamiedakis, A. Hashim, J. Beals, R. V. Penty, and I. H. White, "Low-Cost PCB-Integrated 10-Gb/s Optical Transceiver Built With a Novel Integration Method," IEEE Transactions on Components, Packaging and Manufacturing Technology, vol. 3, pp. 592-600, 2013.

[7]. I. Papakonstantinou, D. R. Selviah, R. Pitwon, and D. Milward, "Low-Cost, Precision, Self-Alignment Technique for Coupling Laser and Photodiode Arrays to Polymer Waveguide Arrays on Multilayer PCBs," IEEE Transactions on Advanced Packaging, vol. 31, pp. 502-511, 2008.

[8]. R. Dangel, et al., "Polymer-Waveguide-Based Board-Level Optical Interconnect Technology for Datacom Applications," IEEE Transactions on Advanced Packaging, vol. 31, pp. 759-767, 2008.

[9]. K. Schmidtke, et al., "960 Gb/s Optical Backplane Ecosystem Using Embedded Polymer Waveguides and Demonstration in a 12G SAS Storage Array," Journal of Lightwave Technology, vol. 31, pp. 3970-3975, 2013.

[10]. F. E. Doany, et al., "Terabit/s-Class Optical PCB Links Incorporating 360-Gb/s Bidirectional $850 \mathrm{~nm}$ Parallel Optical Transceivers," Journal of Lightwave Technology, vol. 30, pp. 560-571, 2012.

[11]. J. Beals, et al., "A terabit capacity passive polymer optical backplane based on a novel meshed waveguide architecture," Applied Physics A: Materials Science \& Processing, vol. 95, pp. 983-988, 2009.

[12]. N. Bamiedakis, R. V. Penty, and I. H. White, "Compact Multimode Polymer Waveguide Bends for Board-Level Optical Interconnects," Journal of Lightwave Technology, vol. 31, pp. 2370-2375, 2013.

[13]. A. Hashim, N. Bamiedakis, R. V. Penty, and I. H. White, "Multimode $90^{\circ}$-Crossings, Combiners and Splitters for a Polymer-Based On-Board Optical Bus," in Conference on Lasers and Electro-Optics (CLEO), pp. 1-2, 2012.

[14]. N. Bamiedakis, et al., "Cost-Effective Multimode Polymer Waveguides for High-Speed On-Board Optical Interconnects," IEEE Journal of Quantum Electronics, vol. 45, pp. 415-424, 2009.

[15]. N. Bamiedakis, A. Hashim, R. V. Penty, and I. H. White, "A $40 \mathrm{~Gb} / \mathrm{s}$ Optical Bus for Optical Backplane Interconnections," Journal of Lightwave Technology, vol. 32, pp. 1526-1537, 2014. 
[16]. J. V. DeGroot Jr., "Cost-effective optical waveguide components for printed circuit applications," in Passive Components and Fiber-based Devices IV (SPIE), pp. 1-12, 2007.

[17]. B. W. Swatowski, et al., "Flexible, stable, and easily processable optical silicones for low loss polymer waveguides," Proc. SPIE 8622, Organic Photonic Materials and Devices XV, pp. 1-11, 2013.

[18]. B. W. Swatowski, C. M. Amb, M. G. Hyer, R. S. John, and W. K. Weidner, "Graded Index Silicone Waveguides for High Performance Computing," in IEEE Optical Interconnects Conference (OIC), pp. 1-3, 2014.

[19]. A. Larsson, "Advances in VCSELs for Communication and Sensing," IEEE Journal of Selected Topics in Quantum Electronics, vol. 17, pp. 1552-1567, 2011.

[20]. P. Westbergh, et al., "High-speed $850 \mathrm{~nm}$ VCSELs operating error free up to $57 \mathrm{Gbit} / \mathrm{s}$," in Electronics Letters(Institution of Engineering and Technology, 2013), pp. 1021-1023.

[21]. D. Kuchta, et al., "64 Gb/s Transmission over 57m MMF using an NRZ Modulated 850nm VCSEL," in Optical Fiber Communication Conference (OFC), pp. 1-3, 2014.

[22]. D. M. Kuchta, et al., "A 71-Gb/s NRZ Modulated 850-nm VCSEL-Based Optical Link," IEEE Photonics Technology Letters, vol. 27, pp. 577-580, 2015.

[23]. N. Bamiedakis, R. V. Penty, I. H. White, P. Westbergh, and A. Larsson, "25 Gb/s Data Transmission over a $1.4 \mathrm{~m}$ Long Multimode Polymer Spiral Waveguide," in Conference on Lasers and Electro-Optics (CLEO), p. paper STu1G.7 2014.

[24]. N. Bamiedakis, et al., "40 Gb/s Data Transmission Over a 1-m-Long Multimode Polymer Spiral Waveguide for Board-Level Optical Interconnects," Journal of Lightwave Technology, vol. 33, pp. 882-888, 2015.

[25]. N. Bamiedakis, J. Chen, R. Penty, and I. White, "Bandwidth Studies on Multimode Polymer Waveguides for $\geq 25$ Gb/s Optical Interconnects," IEEE Photonics Technology Letters, vol. 26, pp. 2004-2007, 2014.

[26]. L. Raddatz, I. H. White, D. G. Cunningham, and M. C. Nowell, "An experimental and theoretical study of the offset launch technique for the enhancement of the bandwidth of multimode fiber links," Journal of Lightwave Technology, vol. 16, pp. 324-331, 1998.

[27]. A. Hashim, N. Bamiedakis, R. V. Penty, and I. H. White, "Multimode Polymer Waveguide Components for Complex On-Board Optical Topologies," Journal of Lightwave Technology, vol. 31, pp. 3962-3969, 2013. 